\title{
Chemo-Immunotherapy with Atezolizumab in Extensive-Stage Small-Cell Lung Cancer; Single-Center Experience
}

\author{
Ahmet Bilgehan SAHIN ${ }^{1}$, Erdem CUBUKCU ${ }^{1}$, Birol OCAK ${ }^{1}$, Adem DELIGONUL ${ }^{1}$, \\ Turgut KACAN ${ }^{2}$, Sibel Oyucu ORHAN ${ }^{1}$, Turkkan EVRENSEL ${ }^{1}$ \\ ${ }^{1}$ Bursa Uludag University, Faculty of Medicine, Department of Medical Oncology \\ ${ }^{2}$ Bursa Yuksek Ihtisas Training and Research Hospital, Department of Medical Oncology, Bursa, TURKEY
}

\begin{abstract}
Chemo-immunotherapy (CIT) with platin, etoposide and monoclonal antibodies targeting the PD-1/PDL-1 pathway has recently improved survival in extensive-stage small-cell lung cancer (SCLC) after decades. We aimed to investigate the efficacy and safety of CIT with atezolizumab in extensive-stage SCLC in chemotherapy naïve patients. Eleven patients who were treated and followed in our center were included in this retrospective observational study. All the patients received carboplatin, etoposide and atezolizumab in the induction phase and atezolizumab in the maintenance phase. The Kaplan-Meier test was used to determine progression-free survival (PFS) and overall survival (OS), and the effects of the sites of metastasis were analyzed using the log-rank test. The median age was 69.9 years, and $81.8 \%$ were male. The median number of CIT and total atezolizumab cycles was 4 and 7 , respectively. 63.6\% received maintenance therapy. Median PFS was 5.2 months (95\% Cl: 3.4-6.9), and median OS was 11.3 months (95\% Cl: 1.0-21.5). The overall response rate was $63.6 \%$. There was no significant difference between patients with and without liver metastasis in terms of PFS and OS. We observed toxicity higher than grade 2 in more than half of the patients, and hematological toxicities were prominent. CIT with carboplatin, etoposide and atezolizumab is efficient and safe in extensive-stage SCLC considering the PFS, OS, response rates, 12-month survival rate, and side effects. The progression of liver lesions was remarkable. Cranial and thoracic radiation are issues that should be discussed in the future with data from clinical studies.
\end{abstract}

Keywords: Atezolizumab, Chemo-immunotherapy, Radiotherapy, Small cell lung cancer

\section{INTRODUCTION}

Lung cancer is the most common and mortal cancer considering its incidence and mortality rate, according to data from GLOBOCAN 2018. ${ }^{1}$ Small cell lung cancer (SCLC) is a neuroendocrine neoplasm that accounts for about $15 \%$ of lung cancers. ${ }^{2}$ SCLC as a clinical entity differs from other types of lung cancer due to its rapid doubling time and early development of the extensive disease, where two-thirds of patients have extensive-stage SCLC at the time of diagnosis. ${ }^{3}$ Standard first-line therapy consists of platinum plus etoposide (PE); although SCLC is sensitive to this combination regimen, relapse of the disease is almost inevitable within the first six months. The median overall survival (OS) is about 10 months, ${ }^{4}$ as next-line treatments are not effective enough when the disease is refractory to the first-line PE. Despite poor outcomes, the PE combination regimen has remained standard firstline therapy for decades.

In the last five years, immunotherapy has become the first-line therapy option for many cancers, including non-small cell lung cancer (NSCLC). For SCLC, immune checkpoint inhibition targeting the programmed cell death 1 (PD-1)/programmed cell death ligand 1 (PD-L1) and cytotoxic T-lymphocyte antigen-4 (CTLA-4) pathways demonstrated antitumor activity but did not prolong survival when used after the first-line PE regimen. ${ }^{5,6}$ 
Recently, first atezolizumab, then durvalumab, which are PD-1/PD-L1 pathways inhibitors, showed significant OS improvement when combined with PE in the trials of IMpower $133^{7}$ and CASPIAN, ${ }^{8}$ respectively. Since this improvement in survival has been achieved for the first time in 30 years, both atezolizumab and durvalumab combined with PE have been accepted as preferred treatments in international guidelines. ${ }^{9}$

Herein, we present the results of a single-center, observational retrospective study with real-life data. We aimed to investigate the efficacy and side effects of atezolizumab in patients with extensivestage SCLC as a first-line therapy combined with PE.

\section{PATIENTS AND METHODS}

\section{Study Population and Data Collection}

We included 11 patients diagnosed with extensivestage SCLC and treated with atezolizumab plus carboplatin-etoposide in the first-line setting at Bursa Uludag University Hospital from January 2019 to May 2020. We retrospectively collected data concerning the clinical characteristics, response, toxicity, and survival from the electronic records of the patients. In each 21-day cycle, carboplatin (area under the curve $5 \mathrm{mg} / \mathrm{mL}$ per min on day 1), etoposide (100 mg/m² on days 1-3) and atezolizumab (1200 mg on day 1) were administered in the induction phase. After completion of 4 to 6 cycles of the induction phase, patients received atezolizumab in the maintenance phase. We continued atezolizumab maintenance therapy in case of evidence of clinical benefits, unless deterioration of ECOG performance, which is attributed to disease progression, was observed. Computed tomography (CT) of the chest, positron emission tomography-computed tomography (PET/CT), and cranial magnetic resonance imaging were performed in all patients for the systemic evaluation of the disease at the time of the beginning of the treatment. PET/CT scan was used to evaluate the response, and thorax CT was added when necessary. Adverse events were graded using the National Cancer Institute Common Terminology Criteria for Adverse Events (version 4.0). Patients without response assessment and those who had missing data about laboratory findings were excluded. Our study was in accordance with the ethical standards of the institutional research committee and with the 1964 Declaration of Helsinki. The clinical research ethics committee of Bursa Uludag University Faculty of Medicine approved the study (Decision No: 2020-6/32).

\section{Outcomes and Statistical Analysis}

Continuous variables were expressed by median (minimum-maximum) values, and categorical variables were expressed by frequency and corresponding percentage values. Response assessment was conducted according to Response Evaluation Criteria in Solid Tumors (version 1.1). Progression-free survival (PFS) was calculated from the beginning of treatment with chemo-immunotherapy (CIT) until disease progression or death from any cause. OS was determined from the time of diagnosis until death from any cause. Life expectancy was analyzed by the Kaplan-Meier method, and the log-rank test was used to investigate the effect of the site of metastasis on survival. The data were statistically processed using the IBM SPSS version 22 software, and a 5\% type-I error level was used for statistical significance.

\section{RESULTS}

A total of 9 (81.8\%) patients were male. The median age of patients was 69.9 years. One patient had large cell neuroendocrine carcinoma presenting brain metastasis. Because the Ki67 of this patient was $70 \%$, he was treated as having SCLC, as recommended by international guidelines. ${ }^{10}$ In total, $90.9 \%$ had de novo metastatic disease. Only one patient had platin sensitive disease, which relapsed after 11 months. Lungs and non-regional lymph nodes were the most common sites of metastasis. Two patients had metastasis in the central nervous system (CNS). The Eastern Cooperative Oncology Group (ECOG) performance status was 0 in $18.2 \%$ of patients, 1 in $72.7 \%$ of patients, and 2 in $9.1 \%$ of patients. In the induction phase, the median number of CIT cycles was four. Following the induction phase, $63.6 \%$ received maintenance therapy, and the median number of overall atezolizumab cycles was 7 (Table 1). 


\begin{tabular}{|c|c|c|c|}
\hline Age, median (min-max) (year) & $69.9(52.5-85.1)$ & & \\
\hline Gender (male/female) (n, \%) & $9 / 2(81.8 \%-18.2 \%)$ & & \\
\hline \multirow[t]{2}{*}{ Histopathology } & SCLC & 10 & (90.9\%) \\
\hline & LCNEC & 1 & $(9.1 \%)$ \\
\hline \multirow[t]{6}{*}{ Sites of metastasis } & Lymph node ${ }^{\alpha}$ & 7 & $(63.6 \%)$ \\
\hline & Lung & 7 & $(63.6 \%)$ \\
\hline & Liver & 4 & (36.4\%) \\
\hline & Bone & 4 & $(36.4 \%)$ \\
\hline & Adrenal gland & 3 & $(27.3 \%)$ \\
\hline & Brain & 2 & $(18.2 \%)$ \\
\hline \multirow[t]{3}{*}{ ECOG performance status score } & 0 & 2 & $(18.2 \%)$ \\
\hline & 1 & 8 & $(72.7 \%)$ \\
\hline & 2 & 1 & $(9.1 \%)$ \\
\hline CIT cycles, median (min-max) & $4(1-6)$ & & \\
\hline Total atezolizumab cycles, median (min-max) & $7(1-19)$ & & \\
\hline Patients received maintenance atezolizumab & 7 (63.6\%) & & \\
\hline \multirow[t]{2}{*}{ Patients received cranial irradiation } & Before treatment & 1 & (9.1\%) \\
\hline & During treatment & 2 & $(18.2 \%)$ \\
\hline \multirow[t]{2}{*}{ Patients received thoracic irradiation } & Before treatment & 1 & (9.1\%) \\
\hline & During treatment & 2 & $(18.2 \%)$ \\
\hline \multirow[t]{5}{*}{ Responses } & Complete response & 1 & $(9.1 \%)$ \\
\hline & Partial response & 6 & (54.5\%) \\
\hline & Overall Response Rate & 7 & (63.6\%) \\
\hline & Stable disease & 3 & $(27.3 \%)$ \\
\hline & Progressive disease & 1 & $(9.1 \%)$ \\
\hline \multirow[t]{2}{*}{ Overall survival rates } & 6-month & $60.6 \%$ & \\
\hline & 12-month & $48.5 \%$ & \\
\hline
\end{tabular}

In the follow-up, seven patients had disease progression, and six patients died. CNS metastasis occurred in two patients under maintenance therapy. Disease progression in the liver was observed in all patients with liver metastasis before treatment. The overall response rate (ORR) was $63.6 \%$. In one patient, a complete response was achieved. Three patients had stable disease, and one progressed. One patient had prophylactic cranial irradiation (PCI) and consolidation thoracic radiotherapy before relapse of the disease. In two patients, cranial irradiation was performed during treatment; in one for cranial metastasis during the induction phase and in the other patient for PCI during the maintenance phase. PCI was planned at the time of data cutoff for one patient. One patient with CNS metastasis at the time of diagnosis refused cranial irradiation. Two patients received consolidative thoracic radiation during treatment in the maintenance phase and we did not observe any adverse effects related to radiation. The clinical courses of each patient are shown in Table 2.

Hematologic toxicity was the most common, while grade 3 and higher hematological toxicity was observed in more than half of patients. None of the patients developed grade 5 treatment-related toxicity. Nausea, the elevation of hepatic enzymes, skin lesions, and acute kidney injury were other adverse events. We did not discontinue treatment in any patients because of toxicity (Table 3 ). 
International Journal of Hematology and Oncology

\begin{tabular}{|c|c|c|c|c|c|c|c|}
\hline & Site of metastasis & $\begin{array}{l}\text { CIT } \\
\text { cycles }\end{array}$ & $\begin{array}{l}\text { MP } \\
\text { cycles }\end{array}$ & $\begin{array}{l}\text { Best } \\
\text { Response }\end{array}$ & $\begin{array}{l}\text { PFS } \\
\text { event }\end{array}$ & $\begin{array}{l}\text { Sites of } \\
\text { progression }\end{array}$ & Clinical course \\
\hline 1 & Liver, lung & 6 & 2 & PR & Yes & $\begin{array}{l}\text { Liver, brain, } \\
\text { bone }\end{array}$ & The patient died after second-line therapy \\
\hline 2 & Brain, lung & 6 & 13 & $\mathrm{CR}$ & No & - & Atezolizumab maintenance is continuing \\
\hline 3 & Bone, liver & 4 & 9 & PR & Yes & Liver, bone & The patient died after second-line therapy \\
\hline 4 & Liver, LN, lung & 1 & 0 & PD & Yes & Liver & $\begin{array}{l}\text { The patient was internalized for AKI and PE } \\
\text { and died. Progression in the liver was ob- } \\
\text { served. }\end{array}$ \\
\hline 5 & $\mathrm{LN}$ & 4 & 14 & PR & No & - & Atezolizumab maintenance is continuing. \\
\hline 6 & Adrenal gland, bone & 5 & 11 & SD & Yes & $\begin{array}{l}\text { Bone, lymph } \\
\text { node }\end{array}$ & $\begin{array}{l}\text { The patients received local therapies } \\
\text { (RT and surgery). Atezolizumab } \\
\text { maintenance is continuing. }\end{array}$ \\
\hline 7 & $\begin{array}{l}\text { Adrenal gland, bone, } \\
\text { liver, LN, lung }\end{array}$ & 4 & 2 & PR & Yes & $\begin{array}{l}\text { Liver, brain, } \\
\text { bone }\end{array}$ & The patient died after second-line therapy \\
\hline 8 & $L N$, lung & 4 & 3 & PR & No & - & Atezolizumab maintenance is continuing. \\
\hline 9 & Brain, LN, lung & 2 & 0 & SD & Yes & $\begin{array}{l}\text { Lung, lymph } \\
\text { nodes }\end{array}$ & $\begin{array}{l}\text { After two cycles of CIT, the patient refused } \\
\text { to receive therapy and died }\end{array}$ \\
\hline 10 & $\begin{array}{l}\text { Adrenal gland, bone, } \\
\text { LN }\end{array}$ & 4 & 0 & SD & Yes & - & $\begin{array}{l}\text { Fifteen days after the } 4 \text { th CIT cycle, the } \\
\text { patient had a myocardial infarction } \\
\text { and died }\end{array}$ \\
\hline 11 & Bone, LN, lung & 5 & 0 & PR & No & - & Atezolizumab maintenance was planned \\
\hline
\end{tabular}

At a median follow up of 9.5 months (95\% CI: 5.812.7), median PFS was 5.2 months (95\% CI: 3.46.9), and median OS was 11.3 months (95\% CI: 1.0-21.5) (Figure 1A, B). A significant difference with respect to PFS and OS was not observed in patients with liver metastasis before treatment $(\mathrm{p}=$ 0.27 and $\mathrm{p}=0.08$, respectively).

\section{DISCUSSION}

To our knowledge, the present retrospective study of small series of patients treated in a single center is the first study reporting real-life experience with CIT with atezolizumab in extensive-stage SCLC in the literature. We focused on the efficacy and tolerability of the treatment. In our study, PFS and OS

\begin{tabular}{|llll|}
\hline Table 3. Adverse events & \multicolumn{3}{l|}{} \\
\hline Event & Any Grade & Grade 1-2 & Grade 3-4 \\
\hline Anemia & $7(63.6 \%)$ & $4(36.4 \%)$ & $3(27.3 \%)$ \\
Neutropenia & $7(63.6 \%)$ & $2(18.2 \%)$ & $5(45.5 \%)$ \\
Thrombocytopenia & $5(45.5 \%)$ & $2(18.2 \%)$ & $3(27.3 \%)$ \\
Nausea & $4(36.4 \%)$ & $4(36.4 \%)$ & - \\
Skin toxicity & $2(18.2 \%)$ & $2(18.2 \%)$ & - \\
Febrile Neutropenia & $1(9.1 \%)$ & - & - \\
Acute kidney injury & $1(9.1 \%)$ & $1(9.1 \%)$ & - \\
Hepatic enzymes elevation & $1(9.1 \%)$ & $1(9.1 \%)$ & $-1 \%)$ \\
\hline
\end{tabular}




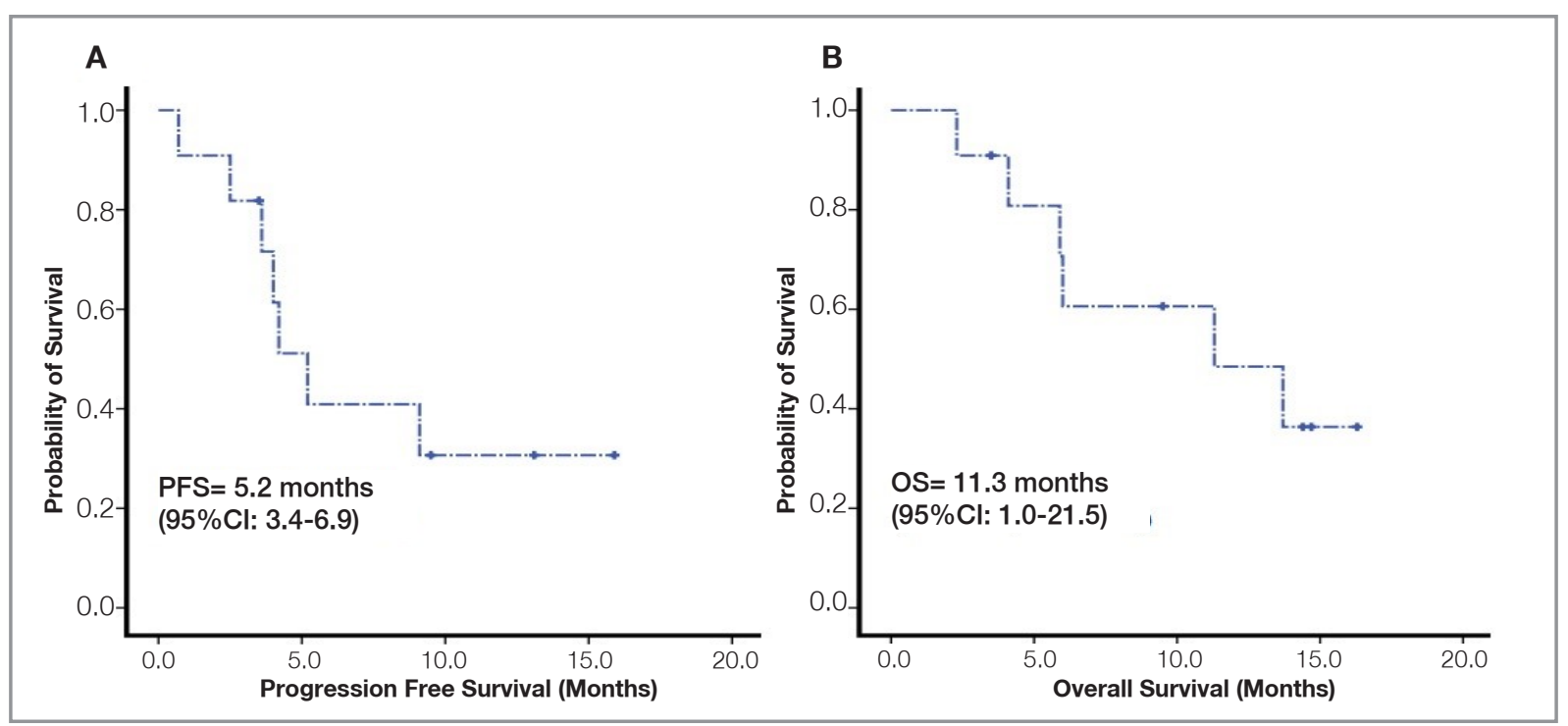

Figure 1. Progression-free survival (PFS) (A) and overall survival (OS) (B) of patients

were determined as 5.2 and 11.3 months, respectively. PFS was similar, and OS was relatively low compared to the Impower $133^{7}$ study, in which PFS and OS were 5.2 and 12.3 months, respectively. In our study, the patients were older than the patients in IMpower 133. Also, the ratio of patients with brain metastasis is approximately twice that of the IMpower133 study. These factors may cause shorter OS in our study.

The ORR was $63.6 \%$ in our study, which is slightly higher than that in the IMpower133 study (ORR: $60.2 \%$ ). The median number of CIT and atezolizumab cycles was similar to that in IMpower133. Likewise, the 12-month survival rate was similar (48.5\% in our study to $51.7 \%$ in IMpower133).

In immunotherapy, predictive biomarkers, such as PDL-1 expression and tumor mutation burden (TMB) in NSCLC and other solid tumors, are investigated. Although PD-L1 expression is reported as uncommon in SCLC, ${ }^{11}$ TMB is known to be high. ${ }^{12}$ However, it is shown that both PD-L1 expression and TMB had no predictive value in subgroup analyses of atezolizumab and durvalumab studies. ${ }^{13,14}$ It is known that there are durable responses in patients benefiting from immunotherapy, and the number of atezolizumab cycles in our four patients was 13 and above. Distinguishing patients showcasing the clinical benefits of immunotherapy from others would guide clinicians not to give up immunotherapy immediately. Markers that can predict response are crucial to both use local therapies more aggressively in the management of patients in whom a response is achieved and protect non-responder patients from the side effects of immunotherapy. Therefore, there remains a need for predictive markers in the immunotherapy of SCLC.

In immunotherapy, liver metastasis is associated with a reduced response and shortened survival. ${ }^{15}$ In CIT studies with atezolizumab, a significant difference was not observed in the comparison of subgroup analyses in terms of liver metastasis in SCLC and NSCLC. ${ }^{7,16}$ In our study, there were three partial responses, and one stable disease among patients with liver metastasis, and PFS and OS did not differ between groups. However, we observed progression in the liver in all patients with liver metastasis before treatment. Likewise, during maintenance atezolizumab, CNS metastasis developed in two patients without CNS metastasis. Both patients did not receive cranial irradiation. In the updated report $^{13}$ of the IMpower133 trial presented in the European Society of Medical Oncology Congress 2019 , OS was longer in the placebo group than in the atezolizumab group, but there was no statistically significant difference in patients with CNS metastasis. Therefore, cranial irradiation may be considered in patients receiving maintenance treat- 
ment. In both subgroups, further studies are needed to investigate the efficacy of maintenance and progression patterns of the disease.

Thoracic radiation was not permitted before and during CIT in both the IMpower 133 and CASPIAN $^{8}$ trials. In our study, three patients received thoracic radiation as consolidative therapy. Of the three patients, one had consolidation radiotherapy before relapse of the disease, approximately one year before CIT and two received during the maintenance phase. We did not observe side effects related to radiation in the patients. It was reported that radiotherapy (RT) might improve the efficacy of immunotherapy with a direct and abscopal effect. ${ }^{17}$ However, the literature lacks randomized controlled trials investigating the efficacy and safety of CIT plus RT in extensive-stage SCLC.

In terms of toxicity, adverse events were more common, especially hematological toxicities. This may be related to the greater presence of older patients in our study than in the reference studies. In more than half of patients, we observed toxicity higher than grade 2. However, no adverse events led to death or treatment discontinuation.

The main limitation of our study is the small sample size. We could not perform further subgroup analysis in patients with cranial metastasis and patients receiving thoracic radiation. In addition, toxicity data were partially limited to laboratory findings due to retrospective design.

In conclusion, in our small series of patients, induction with PE plus atezolizumab and maintenance with atezolizumab is efficient and welltolerated in extensive-stage SCLC considering the PFS, OS, response rates, 12-month survival rate, and side effects. However, the discovery of predictive biomarkers is crucial. Further randomized trials and real-world data are needed to investigate the efficacy of the maintenance immunotherapy in patients with liver and CNS metastasis and clarify the concurrent or subsequent thoracic radiation.

\section{REFERENCES}

1. Bray F, Ferlay J, Soerjomataram I, et al. Global cancer statistics 2018: GLOBOCAN estimates of incidence and mortality worldwide for 36 cancers in 185 countries. CA Cancer J Clin 68: 394-424, 2018.
2. Oronsky B, Reid TR, Oronsky A, Carter CA. What's New in SCLC? A Review. Neoplasia (United States) 19: 842-847, 2017.

3. Früh M, De Ruysscher D, Popat S, et al. Small-cell lung cancer (SCLC): ESMO clinical practice guidelines for diagnosis, treatment and follow-up. Annals of Oncology 24: 99-105, 2013.

4. Farago AF, Keane FK. Current standards for clinical management of small cell lung cancer. Transl Lung Cancer Res 7: 69-79, 2018.

5. Taylor M, Antonia S, Bendell J, et al. Phase I / II study of nivolumab with or without ipilimumab for treatment of recurrent small cell lung cancer (SCLC): CA209-032. J Immunother Cancer 3: 376, 2015.

6. Chung HC, Piha-Paul SA, Lopez-Martin J, et al. Pembrolizumab after two or more lines of previous therapy in patients with recurrent or metastatic SCLC: Results from the KEYNOTE-028 and KEYNOTE-158 Studies. J Thorac Oncol 15: 618-627, 2020.

7. Horn L, Mansfield AS, Szczesna A, et al. First-line atezolizumab plus chemotherapy in extensive-stage small-cell lung cancer. N Engl J Med 379: 2220-2229, 2018.

8. Paz-Ares L, Dvorkin M, Chen Y, et al. Durvalumab plus platinum-etoposide versus platinum-etoposide in first-line treatment of extensive-stage small-cell lung cancer (CASPIAN): a randomised, controlled, open-label, phase 3 trial. Lancet 394: 1929-1939, 2019.

9. National Comprehensive Cancer Network. Small Cell Lung Cancer (Version 3.2020). http://www.nccn.org/professionals/ physician_gls/pdf/sclc.pdf (Access date: 05/02/2020).

10. National Comprehensive Cancer Network. Neuroendocrine and Adrenal Tumors (Version 1.2019). https://www.nccn. org/professionals/physician_gls/pdf/neuroendocrine.pdf (Access date: 05/03/2020).

11. Oizumi S, Yoh K, Matsumoto S, et al. Assessment of PD-L1 expression and oncogenic gene status in patients with smallcell lung cancer: Immuno-oncology biomarker study in LCSCRUM-Japan. J Clin Oncol 37: 8558, 2019.

12. George J, Lim JS, Jang SJ, et al. Comprehensive genomic profiles of small cell lung cancer. Nature 524: 47-53, 2015.

13. Reck M, Liu SV, Mansfield AS, et al. IMpower133: Updated overall survival (OS) analysis of first-line (1L) atezolizumab (atezo) + carboplatin + etoposide in extensive-stage SCLC (ESSCLC). Ann Oncol 30: 710-711, 2019.

14. Paz-Ares L, Goldman JW, Garassino MC, et al. PD-L1 expression, patterns of progression and patient-reported outcomes (PROs) with durvalumab plus platinum-etoposide in ES-SCLC: Results from CASPIAN. Ann Oncol 30: 928-929, 2019.

15. Tumeh PC, Hellmann MD, Hamid O, et al. Liver metastasis and treatment outcome with anti-PD-1 monoclonal antibody in patients with melanoma and NSCLC. Cancer Immunol Res 5: 417-424, 2017. 
16. Socinski MA, Jotte RM, Cappuzzo F, et al. Atezolizumab for first-line treatment of metastatic nonsquamous NSCLC. N Engl J Med 378: 2288-2301, 2018.

17. Nesbit EG, Leal TA, Kruser TJ. What is the role of radiotherapy for extensive-stage small cell lung cancer in the immunotherapy era? Transl Lung Cancer Res 8: 153-162, 2019.

\section{Correspondence:}

\section{Dr. Ahmet Bilgehan SAHIN}

Bursa Uludag Universitesi, Tip Fakultesi

Tibbi Onkoloji Anabilim Dali

16059, Gorukle, Nilufer,

BURSA / TURKEY

Tel: $\quad$ (+90-224) 2951324

e-mail: absahin@uludag.edu.tr dr.absahin@icloud.com

\section{ORCIDs:}

Ahmet Bilgehan Sahin:

Erdem Cubukcu:

Birol Ocak:

Adem Deligonul:

Turgut Kacan:

Sibel Oyucu Orhan:

Turkkan Evrensel:
0000-0002-7846-0870

0000-0002-0070-0889

0000-0001-7537-1699

0000-0002-3669-6391

0000-0002-2067-1560

0000-0001-8217-3471

0000-0002-9732-5340 